\title{
Corrigendum
}

Diabetes Metab J 2021;45:974

https://doi.org/10.4093/dmj.2021.0304

pISSN 2233-6079 · eISSN 2233-6087

Corrigendum: IRB No.

\section{Clinical Significance of Body Fat Distribution in Coronary Artery Calcification Progression in Korean Population}

\author{
Heesun Lee ${ }^{1,2}$, Hyo Eun Park ${ }^{1,2}$, Ji Won Yoon ${ }^{2,3}$, Su-Yeon Choi ${ }^{1,2}$ \\ ${ }^{1}$ Division of Cardiology, Seoul National University Hospital Healthcare System Gangnam Center, Seoul, \\ ${ }^{2}$ Department of Internal Medicine, Seoul National University College of Medicine, Seoul, \\ ${ }^{3}$ Division of Endocrinology, Seoul National University Hospital Healthcare System Gangnam Center, Seoul, Korea
}

Diabetes Metab J 2021;45:219-230. https://doi.org/10.4093/dmj.2019.0161

IRB No. described in the Methods section of the current manuscript has an error. IRB No. should be changed to H-1605-154-768 from H-0907-045-286.

We apologize for any inconvenience that this may have caused. 